\title{
Error Reduction Algorithm for the Generation of a Kinoform with Laser Diode Array
}

\author{
Takayuki Yokoyamaa, ${ }^{\mathrm{a},}$, Yosuke Koga ${ }^{\mathrm{a}}$, Shiyuan Yang ${ }^{\mathrm{a}}$ \\ ${ }^{a}$ Kyushu Institute of Technology, 1-1 Sensui-cho Tobata-ku Kitakyushu-shi Fukuoka-ken, 804-8550, Japan \\ *Corresponding Author: k108126t@ mail.kyutech.jp
}

\begin{abstract}
In recent years, technologies related to laser has been advances, processing technology using the laser has been used in a variety of situations. In this case, the laser diode is superior to other lasers in luminous efficacy. But the power of laser diode is lower. So we use a laser diode array module to obtain the necessary power for laser processing. In addition, shaping the wavefront of the lasers is ideal in the laser processing. In this paper, we describe error reduction algorithm for the Generation of a Kinoform with Laser Diode Array. Kinoform which is a phase type Computer-generated hologram that does not modulate the amplitude of the light, the utilization efficiency of the light can be improved. Finally, this study can be expected to obtain the intensity necessary for laser processing by controlling the intensity distribution of the laser diode array with Kinoform produced by error reduction algorithm.
\end{abstract}

Keywords: Kinoform, Computer-generated hologram, Fourier transform, Laser diode array.

\section{Introduction}

In recent years, the technology of the laser has advanced. And the processing technique of applying the laser is used in various situations. In particular, we can obtain a high power that is required for the laser processing by using a gas laser or a solid-state laser. However, the luminous efficacy of these lasers is lower than laser diode. Therefore, we propose the use of a laser diode.

The disadvantage of the laser diode is that the output is lower than the output of a gas laser or a solid-state laser. On the other hand, the advantage of the laser diodes is that a high luminous efficacy. Moreover, we can obtain a high output by using a lot of laser diodes because the laser diode is small size and can be integrated.

Generally, the laser has a Gaussian distribution as the intensity of the light becomes maximum at the center and is weak at the peripheral portion. Beam shaping of output light is necessary to increase the quality of application. As the method of this beam shaping, there is a method of using a Computer-generated hologram.

The holograms are recorded amplitude and phase information of the light wave. First, we divided into two laser beams in the optical holography. Second, we irradiated one of the light on the object. And we record the interference pattern of the scattered object beam and the other light in the light-sensitive material. To calculate the diffraction pattern in the computer is a computer-generated holography.

Computer-generated hologram is obtained by recording information that has been calculated by a computer generated holography. Unlike an optics hologram, we can reconstruct the arbitrary intensity distribution of light because recording of Computer-generated hologram doesn't need the model in reality. But recording both the amplitude and phase information calculated by the computer generated holography in the recording medium is difficult. So Computer-generated hologram is recorded one of the information either amplitude or phase. Computer-generated hologram can be classified into two types of the amplitude and phase Computer-generated hologram depending on the recorded information. Amplitude Computer-generated hologram is recorded only the amplitude information. It is easy to create a hologram. However, the utilization efficiency of the light is low. Phase Computer-generated hologram is recorded only the phase information. The reconstructed image error increases because the phase Computer-generated hologram does not have the amplitude information. On the other hand, the 
utilization efficiency of the light is high since the light is not absorbed in principle. We use the Kinoform which is a phase Computer-generated hologram as an optical device for shaping the beam because the utilization efficiency of the light is higher than

\section{The method for generation of a Kinoform with laser diode array}

\subsection{Fourier transform type Computer-generated Hologram}

Figure 1 shows Fourier transform type hologram in the case of the single coherent light. The reconstructed image is projected by screen that left the focus distance through a focus distance remote lens from Kinoform. In this case, the light in the reconstructed image area and light in the Kinoform area are relationship of the Fourier transform pair by function of lens.

Information of Kinoform is obtained by inverse Fourier transformation of the reconstructed image. In addition, information of the reconstructed image is obtained by Fourier transformation of Kinoform. Computer-generated hologram is optimized by this principle.

\subsection{Fourier iterative algorithm}

Gerchberg-Saxton algorithm is known as one of the Fourier iterative algorithm repeats the Fourier transform and inverse Fourier transform. First, the distribution with complex amplitude is Fourier-transformed. Second, it is transposed other amplitude distribution which satisfy certain constraints, and the distribution is inverse Fourier-transformed. Finally, it is transposed amplitude distribution which was input first, and this algorithm return to the first calculation again. Thus this algorithm optimize

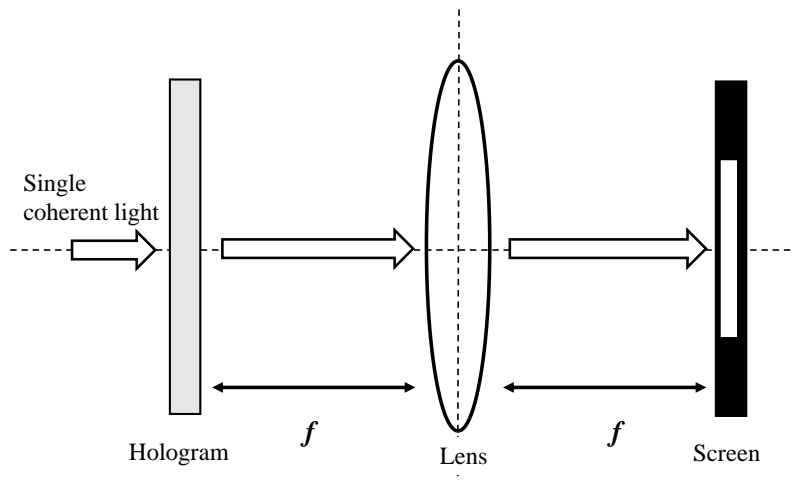

Fig. 1. Fourier transform type hologram.

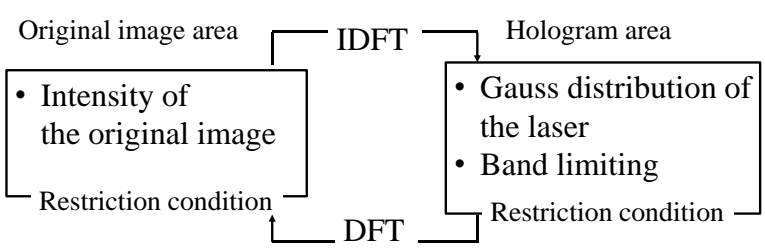

Fig. 2. Fourier iterative algorithm.

the intensity distribution of the reconstructed image which is similar to the intensity distribution of the input image.

\subsection{Phase type Computer-generated Hologram}

When recording the hologram, it is desirable that only one of the information of the amplitude or phase. In this case, the hologram only the phase information is recorded is called phase type hologram. In particular, the phase type computer-generated hologram is called Kinoform.

\section{$2.4 \quad$ Iterative dummy area method}

The reconstructed image errors occur because it is necessary to a constant amplitude distribution in the Kinoform. Iterative dummy area method has been proposed to reduce the error and promote the convergence of the solution. This method can utilize the amplitude and phase freedom of the dummy area by addition of the dummy area that initial value is zero around the original image. In addition, we limit the band in the area of the hologram as restraint condition in iterative dummy area method. Concretely, we set the value of the amplitude and phase to zero at out-of-band area in hologram side. This condition prevent aliasing error by satisfy the sampling theorem in computer-generated hologram.

\subsection{Controlling the intensity distribution of laser diode array}

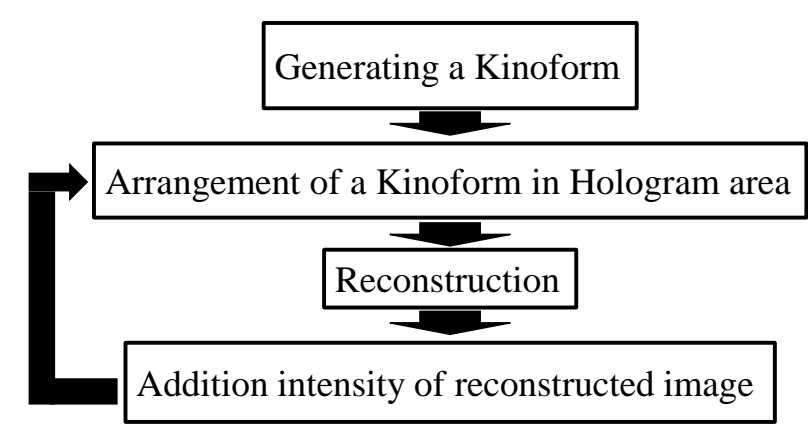

Fig. 5. Flow chart of the calculation. 


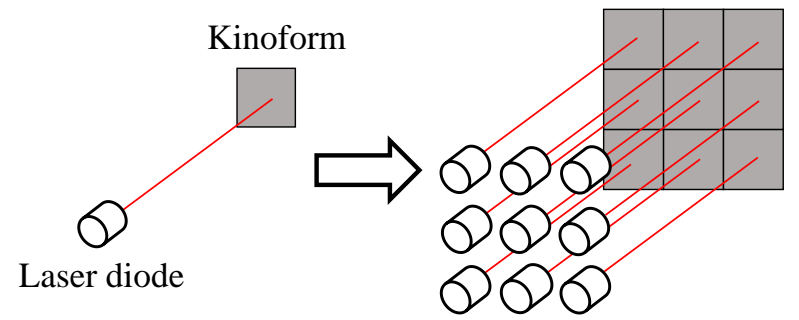

Fig. 6. Arrangement of a Kinoform.

Using the principle that we showed by the above, we generate a Kinoform. We reproduce this Kinoform as many the number of lasers and locates it so that pixels of each Kinoform are not piled up. Then, we make a central coordinate of the optical axis of each laser source of the laser diode array and each Kinoform agree. As shown Fig.6, we locate Kinoforms reproduced so that surround the Kinoform which we located in the center of the dummy area and reconstruct it. The reconstruction is Fourier transform of phase distribution of each Kinoform and the intensity distribution of a supporting laser. If the intensity and phase distribution is the same even if coordinates of the point of the Kinoform are different, the intensity distribution of the reconstructed image does not change by the shift rule. The shift rule shown by function (2) is one of the properties of the Fourier transform. Then, we define a and $b$ are any fixed numbers, $G\left(u_{v} v\right)$ as distribution of a Kinoform, and $g(x, y)$ as distribution the reconstructed image. We can understand that a change in the exponent only by function (2). In other words, we can't recognize only changing of the phase distribution in the reconstructed image.

$$
\begin{aligned}
& \mathcal{F}\left[G\left(u_{v} v\right)\right]=g\left(x_{v} y\right) \\
& \mathcal{F}\left[G\left(u-a_{v} v-b\right)\right]=g\left(x_{v} y\right) e^{-j 2 \pi(x a+y b)}
\end{aligned}
$$

Also, we know coherence of the laser each other is small. Therefore, we add the intensity distribution in the reconstructed image from each Kinoform. In this way, we can control the intensity distribution of the reconstructed image which we expect the intensity distribution of light of the laser diode array by Kinoform.

\section{Error reduction algorithm for the generation of a Kinoform with laser diode array}

We describe error reduction algorithm for the Generation of a Kinoform with Laser Diode Array. First, we design a Kinoform with a single Laser Diode Array. We perform simulation that reconstruct with the Kinoform to

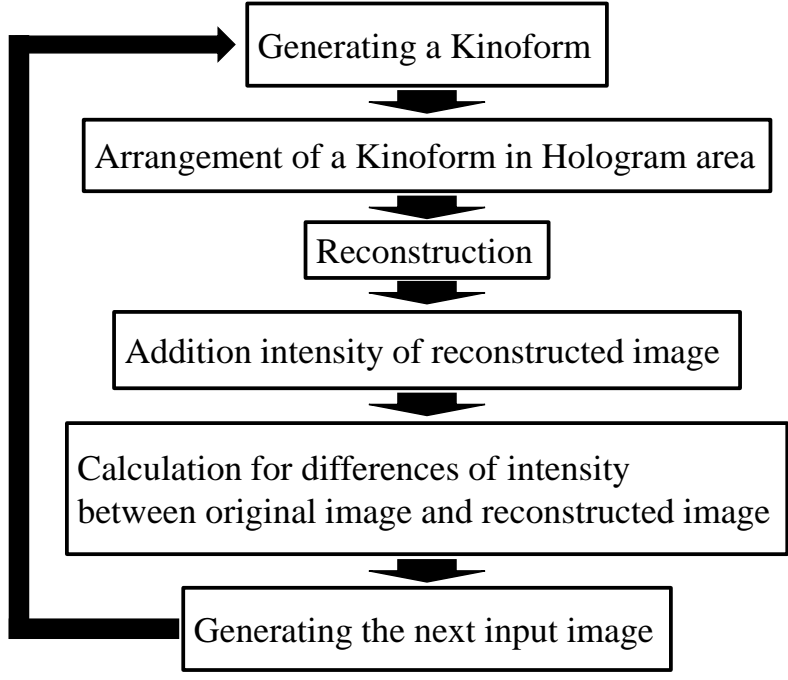

Fig. 7. Flow chart of Error Reduction Algorithm.

obtain reconstructed image. We calculate that difference of the intensity distribution between reconstructed image $g_{1}\left(x_{v} y\right)$ and original image $g_{0}\left(x_{x} y\right)$ with function (3).

$$
\Delta I_{n}\left(x_{x} y\right)=\left|g_{0}\left(x_{x} y\right)\right|^{2}-(\mathrm{n}+1)-\sum_{k=1}^{n}\left|g_{k}\left(x_{x} y\right)\right|^{2}
$$

Then, we define $\mathrm{n}$ as light source number. We define the intensity distribution $\Delta I_{n}$ as the original image to design a Kinoform with the second light source. By repeating the above procedure, we design the plural Kinoforms of the number same as the light sources. Then we arrange these Kinoforms into array form in the same way as Fig.6. Fig.7 shows this process.

\section{Simulation and results}

We simulate under the following conditions.

- Image size is $128 \times 128$ pixels

- The light source number is 9

- Dummy area size is 4 times from original image size

- Band limiting is $1 / 2$

We input the square intensity distribution such as Fig. 8 as an original image, and we define the intensity distribution which added dummy area to original image as the input signal. In this chapter, we compare reconstructed image by Kinoform designed without error reduction algorithm with reconstructed image by Kinoform designed with error reduction algorithm. In addition, we confirm a case that changed the repetition number of times of Fourier iterative algorithm. 
Fig.9 (a) shows an image which is reconstructed by Kinoform designed without error reduction algorithm, and Fig.9 (b) shows an image which is reconstructed by Kinoform designed with error reduction algorithm. Then, the repetition number of times of Fourier iterative algorithm is 200 times. Fig.9 (a) has a large error of intensity distribution in this lower-right but this error are reduced in Fig.9 (b).

In addition, Fig.10 (a) and Fig.10 (b) are reconstructed by Kinoform that the repetition number of times of Fourier iterative algorithm is 2000 times. Fig.10 (b) is reconstructed by Kinoform designed with error reduction algorithm and Fig.10 (a) is not used. The reconstructed image error is reduced by the repetition number of times of Fourier iterative algorithm increase in both cases. Fig.11 is Fig.10 which was extended and performed trimming so that it is easy to compare these images. Even in this case, Fig.11 (a) has a few error of intensity distribution but this error are

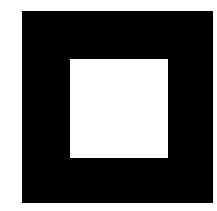

Fig. 8. Original image.

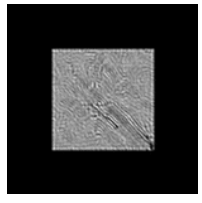

(a)

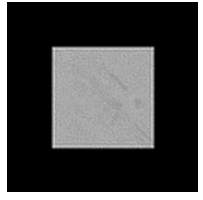

(b)
Fig. 9. Reconstructed images (200 times iteration).

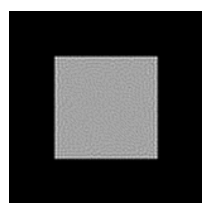

(a)

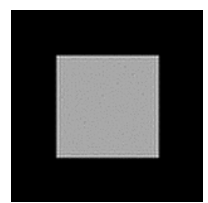

(b)
Fig. 10. Reconstructed images (2000 times iteration).

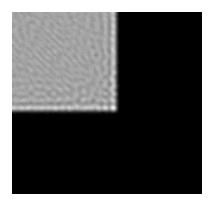

(a)

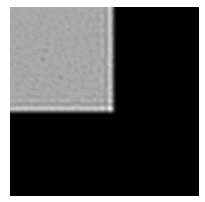

(b)
Fig. 11. Expanded images. reduced in Fig.11 (b).

\section{Conclusions}

In this study, we showed that it is possible to control the intensity distribution of light of laser diode array. In addition, we confirmed that the Kinoform produced Error Reduction Algorithm reduce the reconstructed image errors. As a result, the reconstructed image became better by increase the repetition number of times of Fourier iterative algorithm. Error Reduction Algorithm is effective when the repetition number of times of Fourier iterative algorithm is many.

\section{References}

(1) Takayuki Yokoyama, Yosuke Koga, Yuki Misaki, and Shiyuan Yang : "Method for Controlling the Intensity Distribution of Laser Diode Array Using Computer-generated Hologram", Proceedings of the $3^{\text {rd }}$ International Conference on Industrial Application Engineering, 2015

(2) Toshinori Hora, and Shiyuan Yang : "Iterative Dummy Area Method with Flexible Dummy Area Size for the Design of Kinoform", Journal of the Institute of Industrial Applications Engineers, Vol. 1, No. 2, pp. 69-72, 2013

(3) R. W. Gerchberg, and W. O. Saxton, Optik 35, 37, 1972

(4) Shiyuan Yang, and Hiroaki Takajo : "Speckle Reduction of Kinoform Reconstruction Utilizing the $2 \pi$ Ambiguity of Image Phase Differences", Optical Review, Vol. 12, No. 2, 93-96, 2005 